\title{
Oleanolic Acid Induces the Type III Secretion System of Ralstonia solanacearum
}

\author{
Dousheng Wu ${ }^{1+}$, Wei Ding ${ }^{1 *}$, Yong Zhang ${ }^{2}$, Xuejiao Liu' ${ }^{1}$ and Liang Yang ${ }^{1}$ \\ ${ }^{1}$ Laboratory of Natural Products Pesticides, College of Plant Protection, Southwest University, Chongqing, China, ${ }^{2}$ Research \\ Center of Bioenergy and Bioremediation, College of Resources and Environment, Southwest University, Chongaing. China
}

OPEN ACCESS

Edited by:

Maria Tereza Dos Santos Correia,

Universidade Federal

de Pernambuco, Brazil

Reviewed by:

llaria Braschi,

University of Bologna, Italy

Carmen R. Beuzón,

Universidad de Málaga, Spain

${ }^{*}$ Correspondence:

Wei Ding

dwing818@163.com

${ }^{\dagger}$ Present address:

Dousheng Wu,

Department of General Genetics,

Centre for Plant Molecular Biology, Eberhard-Karls-University Tuebingen,

Auf der Morgenstelle 32,

72076 Tuebingen, Germany

Specialty section:

This article was submitted to

Antimicrobials, Resistance

and Chemotherapy,

a section of the journal

Frontiers in Microbiology

Received: 25 October 2015 Accepted: 07 December 2015 Published: 22 December 2015

Citation:

Wu D, Ding W, Zhang Y, Liu X and Yang L (2015) Oleanolic Acid Induces the Type III Secretion System

of Ralstonia solanacearum.

Front. Microbiol. 6:1466.

doi: 10.3389/fmicb.2015.01466
Ralstonia solanacearum, the causal agent of bacterial wilt, can naturally infect a wide range of host plants. The type III secretion system (T3SS) is a major virulence determinant in this bacterium. Studies have shown that plant-derived compounds are able to inhibit or induce the T3SS in some plant pathogenic bacteria, though no specific T3SS inhibitor or inducer has yet been identified in $R$. solanacearum. In this study, a total of 50 different compounds were screened and almost half of them (22 of 50) significantly inhibited or induced the T3SS expression of $R$. solanacearum. Based on the strong induction activity on T3SS, the T3SS inducer oleanolic acid (OA) was chosen for further study. We found that OA induced the expression of T3SS through the HrpGHrpB pathway. Some type III effector genes were induced in T3SS inducing medium supplemented with OA. In addition, OA targeted only the T3SS and did not affect other virulence determinants. Finally, we observed that induction of T3SS by OA accelerated disease progress on tobacco. Overall our results suggest that plant-derived compounds are an abundant source of $R$. solanacearum T3SS regulators, which could prove useful as tools to interrogate the regulation of this key virulence pathway.

Keywords: $R$. solanacearum, type III secretion system, plant-derived compounds, oleanolic acid, induction

\section{INTRODUCTION}

Ralstonia solanacearum is a Gram-negative soil-borne bacterial plant pathogen that infects more than 200 plant species from over 50 families, including agriculturally and economically important crops such as tobacco, potato, eggplant, and tomato, in tropical and subtropical regions of the world (Genin, 2010). Bacterial wilt caused by this bacterium leads to serious economic losses to agricultural production every year. $R$. solanacearum enters host roots from the soil, colonizes the plant vasculature, and produces a large amount of extracellular polysaccharides (EPS), resulting in the wilting and death of host plants (Schell, 2000).

Like many other plant and animal pathogenic bacteria, R. solanacearum depends on the type III secretion system (T3SS) to invade its hosts. This specialized needle-like delivery machine, encoded by a cluster of about 20 hypersensitive response and pathogenicity ( $h r p$ ) genes, can inject effector proteins directly into host cells suppressing plant innate immunity or activating effector-triggered immunity (Cunnac et al., 2004; Jones and Dangl, 2006). In R. solanacearum, the regulatory cascade linking the expression of T3SS to host contact has been well characterized. The initial activation of hrp genes is triggered upon recognition of a cell wall component by the outer membrane receptor PrhA, which transfers the plant cell interaction dependent signals through a complex signal cascade 
PrhA-PrhR/PrhI-PrhJ-HrpG (Brito et al., 1999, 2002; Aldon et al., 2000). HrpG, a two-component response regulator of the OmpR subfamily, is a key regulator in this cascade. Transcription of HrpG is regulated by both the above well-studied pathway and a PhcA-dependent pathway (Brito et al., 1999; Genin et al., 2005). Downstream of the central regulator HrpG is HrpB, an AraC- family transcriptional activator, which directly controls transcription of the T3SS structural genes and a large repertoire of effector genes (Occhialini et al., 2005). Another regulator that affects the transcription of the HrpB regulon is PrhG and the PrhG-HrpB pathway is regulated by an unrelated virulence operon (Plener et al., 2010; Zhang et al., 2013). Homologs of HrpB and HrpG can be found in Xanthomonas sp. (Wengelnik and Bonas, 1996), while other upper regulators in this cascade are not conserved in other bacterial pathogens.

Since the assembly of the T3SS and the secretion of effector proteins require a lot of energy, pathogens often do not fully express the T3SS until they come into contact with host tissues. T3SS genes in $R$. solanacearum are specifically induced in response to the bacterium-plant cell contact (Aldon et al., 2000). Recent studies also showed that the T3SS regulator $h r p B$ and some $h r p B$-regulated genes are induced in bacteria growing inside infected plants (Jacobs et al., 2012; Monteiro et al., 2012), which is inconsistent with the previous view that $R$. solanacearum T3SS genes are only activated during the early stages of host recognition and bacterial colonization. On the other hand, the expression of T3SS associated genes is regulated by a variety of environmental factors (e.g., $\mathrm{pH}$; growth phase; temperature; nutrition; or cell density) in different ways in different bacteria (Arlat et al., 1992; Wei et al., 1992; Van Dijk et al., 1999; Tang et al., 2006; Stauber et al., 2012). For example, the expression level of T3SS in bacteria is modulated by the carbon source. The best carbon source for $R$. solanacearum hrp genes expression is pyruvate (Arlat et al., 1992), while the optimal carbon inducer for Pseudomonas syringae pathovar tomato DC3000 is fructose (Stauber et al., 2012). Moreover, bacterial T3SS genes are almost inhibited in rich medium but significantly induced in minimal or inducing medium.

In addition to host signals and environmental factors, T3SS genes are also regulated by chemical or natural compounds, which can alter T3SS expression in both animal and plant pathogenic bacteria (Felise et al., 2008; Aiello et al., 2010; Duncan et al., 2012; Yang et al., 2014). This makes the T3SS an attractive target for the development of new agents for disease control. A large-scale screening has identified several classes of T3SS inhibitors, including salicylidene acylhydrazides and thiazolidinone, in animal pathogens (Bailey et al., 2007; Dahlgren et al., 2007; Negrea et al., 2007; Felise et al., 2008; Tree et al., 2009; Veenendaal et al., 2009). Thiazolidinone has further been shown to block the T3SS of the plant pathogen P. syringae (Felise et al., 2008). Recently, some plant phenolic compounds and their derivatives (e.g., p-coumaric acid; benzoic acid; trans-4hydroxycinnamohydroxamic) were found to be able to inhibit the T3SS in plant pathogens Erwinia amylovora or Dickeya dadantii (Li et al., 2009, 2015; Khokhani et al., 2013). Interestingly, some T3SS inducers [e.g., o-coumaric acid; $t$-cinnamic acid; trans-2-(4-hydroxyphenyl)-ethenylsulfonate] were also found for
E. amylovora or D. dadantii (Yang et al., 2008; Khokhani et al., 2013). These phenolic compounds inhibit or induce the T3SS through different signal pathways, even in the same phytopathogenic bacteria. For example, the inhibition of $D$. dadantii T3SS expression by $p$-coumaric acid is moderated through the HrpX/Y two-component system (Li et al., 2009), while $o$-coumaric acid and $t$-cinnamic acid induce the $D$. dadantii T3SS expression through the $r s m B$-RsmA pathway (Yang et al., 2008). Furthermore, the inhibition of T3SS in E. amylovora by exogenous compounds could reduce disease development and T3SS dependent HR (Khokhani et al., 2013; Yang et al., 2014), suggesting it is possible to control plant diseases through the inhibition of T3SS. Although some efforts have been put into the identification of T3SS inhibitors or inducers for plant pathogens, regulation of the T3SS by plant-derived compounds remains largely unknown in most plant pathogenic bacteria.

Given that the T3SS is well conserved in animal and plant pathogenic bacteria, and is able to be either repressed or induced by exogenous compounds in some specific pathogens, we hypothesized that some compounds may alter the expression of T3SS in $R$. solanacearum. In this study, different kinds of compounds were screened for their effect on $R$. solanacearum T3SS expression. The results showed that T3SS expression of this bacterium was either induced or inhibited by some of the screened compounds. Oleanolic acid (OA), one of the best inducers, was further evaluated for its effect on T3SS regulatory components. Furthermore, the effect of $\mathrm{OA}$ on disease development in tobacco was investigated.

\section{MATERIALS AND METHODS}

\section{Bacterial Strains and Growth Conditions}

The R. solanacearum wild-type strain CCT818 (phylotype I, race 1, biovar 3) and the popA-lacZYA reporter strain CCT877 were used in this study. The wild-type strain was originally isolated from an infected tobacco plant in Chongqing, China in 2013. The popA-lacZYA reporter strain was constructed using the recombinant plasmid ppop3 as previously described (Zhang et al., 2011). R. solanacearum was grown in rich B medium or hydroponic plant culture medium supplemented with $2 \%$ sucrose [plant-sucrose (PS) medium] at $28^{\circ} \mathrm{C}$ (Yoshimochi et al., 2009).

\section{Sources and Description of Screened Compounds}

Compounds 1-14, 16-38, and 47-50 were purchased from Shanghai Yuanye Bio-Technology Co., Ltd (Shanghai, China). Compound 15 was extracted and purified from the leaves of Artemisia annua L by our lab members. Compounds 39 to 46 were purchased from Sangon Biotech (Shanghai, China). Among them, compounds 1-38 are natural or plant-derived compounds, which have been reported to have good biological activity in medicine or as pesticides in the field. Compounds 39-46 are major root exudates of tobacco. Compound 47 was previously reported to inhibit T3SS in E. amylovora (Khokhani et al., 2013) and compound 48 was reported to induce the T3SS genes of the plant pathogen D. dadantii (Yang et al., 2008). Compounds 49 
and 50 are widely used plant disease resistance inducers. When screening, compounds were first resolved in dimethyl sulfoxide (DMSO) and a final concentration of $100 \mu \mathrm{M}$ was added to the PS medium.

\section{$\beta$-Galactosidase Activity Assay Based Compounds Screening}

Expression of $p o p A$ was analyzed by determining the $\beta$-galactosidase activity of the lac-ZYA reporter gene. The $\beta$-galactosidase activity assay was performed as previously described with appropriate modifications (Zhang et al., 2015). The popA-lacZYA reporter strain was grown in rich $\mathrm{B}$ broth overnight and transferred to hrp-inducing PS medium supplemented with DMSO or $100 \mu \mathrm{M}$ concentrations of the compounds. PS medium was used to mimic the plant environment and induce the $h r p$ genes expression. When $\mathrm{OD}_{600}$ of the culture suspension reached 0.1 to 0.2 , the $\beta$-galactosidase activity of each treatment were measured. Enzyme activities were calculated as previously described (Zhang et al., 2015). Three replicates were used in each of the independent experiment and each experiment was repeated two times.

\section{Measurement of Growth Curve}

$R$. solanacearum cells were first grown overnight in rich $B$ broth at $28^{\circ} \mathrm{C}$. The bacterial suspension $(\mathrm{OD} 600 \approx 1.0)$ was then transferred into fresh B medium supplemented with DMSO or $100 \mu \mathrm{M}$ of $\mathrm{OA}$ with a proportion of $1: 100$. The growth curve was monitored for $36 \mathrm{~h}$ with a 4 -h interval. The cultures were grown in $250-\mathrm{ml}$ culture flasks, and $3 \mathrm{ml}$ of the cultures were taken at each time point to determine the $\mathrm{OD}_{600}$ value by using the normal spectrophotometer. Three independent experiments were performed and three culture flasks were used for each treatment in each independent experiment.

\section{Biofilm Assay}

To measure biofilm formation in $R$. solanacearum after compounds treatment, the polyvinylchloride (PVC) microtiter plate assay was used as previously described with some modifications (Yao and Allen, 2007). Briefly, overnight cultured bacterial suspension was centrifuged and resuspended in sterile water; the $\mathrm{OD}_{600}$ of the resuspension was exactly adjusted to 0.1 . Then $5 \mu \mathrm{l}$ of the $\mathrm{OD}_{600}$ adjusted suspension was added into $95 \mu \mathrm{l}$ of B medium supplemented with DMSO or different concentrations of $\mathrm{OA}$ and incubated at $30^{\circ} \mathrm{C}$ for $24 \mathrm{~h}$ without shaking. Crystal violet staining and biofilm quantification were performed as previously described (Yao and Allen, 2007) except that the absorbance was determined at $490 \mathrm{~nm}$ using a microplate reader (Bio-Rad).

\section{RNA Extraction and Quantitative Real-Time PCR}

Overnight cultured bacterial suspension was inoculated to fresh PS medium supplemented with DMSO or $100 \mu \mathrm{M}$ concentrations of compounds and shake culture at $28^{\circ} \mathrm{C}$ for 6 h. Bacterial cells were collected by centrifuge. Total RNA was extracted from the collected cells using TRNzol reagent according to the manufacturer's instructions (Tiangen Biotech Co. Ltd, Beijing, China) and then treated with RNase-free DNase I (Tiangen Biotech Co. Ltd, Beijing, China) to remove any genomic DNA contaminations. RNA degradation and contamination were checked on $1 \%$ agarose gels and RNA concentration and purity were monitored using the Nanovue UV-Vs spectrophotometer (GE Healthcare Bio-Science, Uppsala, Sweden). cDNA was synthesized from $1 \mu \mathrm{g}$ of total RNA using the iScript cDNA synthesis kit (Bio-Rad, Hercules, CA, USA).

The primers of tested genes were designed using Primer Blast in NCBI and synthesized by Life Technologies. Sequences of the primers are listed in Supplementary Table S1. The serC gene was used as the reference gene to normalize gene expression (Monteiro et al., 2012). All quantitative real-time PCR (qRTPCR) analyses were performed on the CFX96 Manager (Bio-Rad) in a $20 \mu \mathrm{l}$ reaction system which consisted of $10 \mu \mathrm{l}$ Sso Fast ${ }^{\mathrm{TM}}$ EvaGreen Supermix (Bio-Rad), $1 \mu$ l of diluted cDNA, $0.2 \mathrm{mM}$ of each primer and $7 \mu \mathrm{l}$ of milliQ $\mathrm{H}_{2} \mathrm{O}$. The amplification protocol was as follows: $3 \mathrm{~min}$ at $95^{\circ} \mathrm{C}$, followed by 40 cycles of $95^{\circ} \mathrm{C}$ for $10 \mathrm{~s}$, and $60^{\circ} \mathrm{C}$ for $20 \mathrm{~s}$. After that, a melting curve from 60 to $95^{\circ} \mathrm{C}$ was applied to test the specificity and consistency of the PCR products. Normalized gene expression was calculated by Bio-Rad CFX Manager 3.0 software using the $\Delta \Delta$ Cq method.

\section{Virulence Assay}

Normal soil drenching was used to evaluate the virulence of $R$. solanacearum after OA treatment. R. solanacearum cells were shake culture in PS medium supplemented with DMSO or $100 \mu \mathrm{M}$ of $\mathrm{OA}$ at $28^{\circ} \mathrm{C}$ for $6 \mathrm{~h}$ and bacterial suspension of each treatment was adjusted to the same concentration. Each individual unwounded 6-week old tobacco plant (Yunyan87) was soaked with either DMSO treated or OA treated bacterial suspension to create a final inoculation density of $1 \times 10^{8} \mathrm{CFU} / \mathrm{g}$ soil medium. Inoculated plants were put into the climate room at $28^{\circ} \mathrm{C}$ with $14 \mathrm{~h} / 10 \mathrm{~h}$ light/dark cycle. Disease was recorded daily using a disease index scale of 0-4 (0: no symptoms appeared; 1 : $1-25 \%$ of leaves wilted; 2 : $26-50 \%$ of leaves wilted; $3: 51-75 \%$ of leaves wilted; 4: 76-100\% of leaves wilted). Each treatment contained 16 plants in an independent experiment and the inoculation assay was repeated three times.

\section{Statistical Analysis}

Data were statistically analyzed using a one-way analysis of variance (ANOVA) and Student's $t$-test under the significance level of 0.05 ( $P$-value $=0.05)$ in SPSS 17.0. Disease index significance analysis was performed as previously described using repeated-measures ANOVA (Jacobs et al., 2013).

\section{RESULTS}

\section{A Screen Identifies Oleanolic Acid as a Strong Inducer of T3SS}

In order to screen for compounds that could induce or repress the expression of T3SS in $R$. solanacearum, a T3SS reporter strain was constructed. The lacZYA reporter gene which encodes a variant 
of $\beta$-galactosidase was inserted into the type III effector gene popA. In the popA-lacZYA fusion strain, the lacZYA gene shares the promoter with $p o p A$ whose activity represents the expression level of the downstream T3SS. This reporter strain was cultured in a PS medium, which had a stronger induction on $p o p A$ expression than hrp-inducing minimal medium (Yoshimochi et al., 2009), supplemented with DMSO (solvent of the screened compounds) or $100 \mu \mathrm{M}$ of each compound. The expression level of $p o p A$ was then determined by measuring the $\beta$-galactosidase activity (Table 1). Among the 50 plant-derived compounds screened, six compounds had a strong inhibitory effect on the measured $p o p A$ promoter activity. The expression of $p o p A$ was reduced by more than $50 \%$ of the reference level after stearic acid (compound 41, SA) and benzoic acid (compound 47, BA) treatment at the concentration of $100 \mu \mathrm{M}$. Since SA and BA inhibited the expression of $p o p A$ at a high concentration, we further examined the inhibitory effect of these two compounds at a concentration of $10 \mu \mathrm{M}$. The result showed that $10 \mu \mathrm{M}$ of SA and BA did not result in significant inhibition on popA expression (Figure 1A).
In addition to these inhibitors, however, 16 other compounds showed a significant induction effect on $p o p A$ expression, among which compound 28, OA (Figure 1B), was the best inducer with an 8.1 -fold increase in $\beta$-galactosidase activity at the concentration of $100 \mu \mathrm{M}$ compared to DMSO control. A strong induction effect was also observed when OA was used at a concentration of $10 \mu \mathrm{M}$ (Figure 1A). Although we were initially interested in screening for strong T3SS inhibitor(s), we found that in our experiments OA was a more attractive compound for investigating the regulatory mechanism of $R$. solanacearum T3SS by exogenous compounds because of the strong induction effect.

\section{OA Induces the Expression of T3SS in a Concentration-Dependent Manner}

To further determine the effect of the OA concentration on $p o p A$ expression, we examined the induction effect of this compound at different concentrations, ranging from 6.25 to $100 \mu \mathrm{M}$. Consistent with the initial screening, $100 \mu \mathrm{M}$ of $\mathrm{OA}$ showed an 8.8-fold induction compared to the

TABLE 1 | Expression of Ralstonia solanacearum popA measured by the $\beta$-galactosidase activity of the lacZYA reporter fusion gene in PS medium or PS medium supplemented with plant derived compounds.

\begin{tabular}{|c|c|c|c|c|c|}
\hline Number & Compound ${ }^{a}$ & $\beta$-galactosidase activity (mean $\pm S D)^{b}$ & Number & Compound $^{a}$ & $\beta$-galactosidase activity (mean $\pm \mathrm{SD})^{\mathrm{b}}$ \\
\hline & DMSO & $368 \pm 5.2$ & & DMSO & $194 \pm 10.0$ \\
\hline (1) & Chlorogenic acid & $661 \pm 17.2^{*}$ & (26) & Synephrine & $224.5 \pm 7.5$ \\
\hline (2) & Protocatechuic acid & $430 \pm 2.5^{*}$ & $(27)$ & Curcumol & $185 \pm 17.0$ \\
\hline (3) & Muscone & $388 \pm 21.9$ & (28) & Oleanolic acid & $1580 \pm 34.5^{*}$ \\
\hline (4) & Resveratrol & $467 \pm 4.7^{*}$ & (29) & Arctigenin & $221 \pm 9.5$ \\
\hline (5) & Eugenol & $350 \pm 1.0$ & (30) & Dicoumarolum & $236 \pm 19.5$ \\
\hline (6) & Gastrodin & $363 \pm 3.3$ & (31) & Luteolin & $282 \pm 62.0$ \\
\hline (7) & Cepharanthine & $431 \pm 13.4^{*}$ & (32) & Esculin hydrate & $275 \pm 6.8^{*}$ \\
\hline (8) & Deoxyschizandrin & $394 \pm 14.5$ & (33) & Apigenin & $462 \pm 10.0^{*}$ \\
\hline (9) & Capsaicin & $434 \pm 9.5^{*}$ & (34) & Scopolamine butylbromide & $207 \pm 7.5$ \\
\hline (10) & Icariin & $247 \pm 6.1^{*}$ & (35) & Diosgenin & $180 \pm 7.0$ \\
\hline (11) & Tetrandrine & $410 \pm 28.0$ & (36) & Daphnetin & $204 \pm 8.5$ \\
\hline (12) & Alantolactone & $362 \pm 6.7$ & (37) & Stigmasterol & $201 \pm 10.5$ \\
\hline (13) & Palmitic acid & $284 \pm 14.9^{*}$ & (38) & Glycyrrhetic acid & $156 \pm 2.0^{*}$ \\
\hline \multirow[t]{3}{*}{ (14) } & Citric acid & $425 \pm 52.8$ & & & \\
\hline & & & & DMSO & $186 \pm 11.0$ \\
\hline & DMSO & $310 \pm 4.0$ & (39) & Succinic acid & $259 \pm 5.0^{*}$ \\
\hline (15) & Scopoletin & $452 \pm 31.5^{*}$ & $(40)$ & Fumaric acid & $277 \pm 13.5^{*}$ \\
\hline (16) & Tubeimoside & $375 \pm 8.5^{*}$ & $(41)$ & Stearic acid & $86 \pm 9.5^{*}$ \\
\hline (17) & Xanthotoxin & $324 \pm 24.5$ & $(42)$ & L-Malic acid & $396 \pm 37.5^{*}$ \\
\hline (18) & Bergeninum & $352 \pm 4.0^{*}$ & (43) & L-Tryptophan & $190 \pm 14.5$ \\
\hline (19) & Lappaconitine & $314 \pm 13.0$ & $(44)$ & L-Histidine & $194 \pm 10.5$ \\
\hline (20) & Taxifolin & $786 \pm 28.0^{*}$ & $(45)$ & L-valine & $211 \pm 16.0$ \\
\hline (21) & Podophyllotoxin & $389 \pm 3.5^{*}$ & $(46)$ & L-Arginine & $212 \pm 5.5$ \\
\hline (22) & Cryptotanshinone & $344 \pm 56.5$ & $(47)$ & Benzoic acid & $73 \pm 8.5^{*}$ \\
\hline (23) & Vindoline & $319 \pm 32.0$ & (48) & Trans-Cinnamic acid & $164 \pm 8.0$ \\
\hline (24) & Emodin & $214 \pm 12.5^{*}$ & (49) & Methyl Jasmonate & $193 \pm 9.6$ \\
\hline (25) & Patchouli alcohol & $365 \pm 24.5$ & (50) & Salicylic acid & $212 \pm 11.0$ \\
\hline
\end{tabular}

aPS medium (plant-sucrose medium) was supplemented with DMSO or $100 \mu \mathrm{M}$ of the indicated compounds. The compounds were assayed two times.

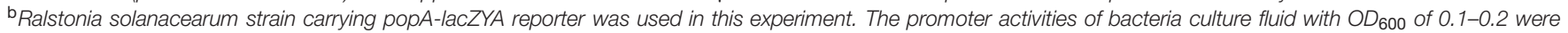

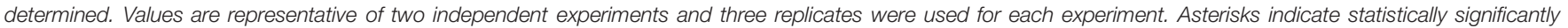

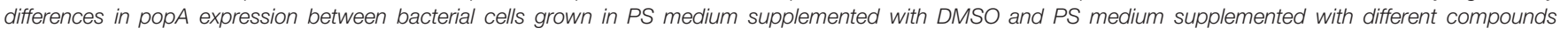
$(P<0.05$, Student's t-test). 


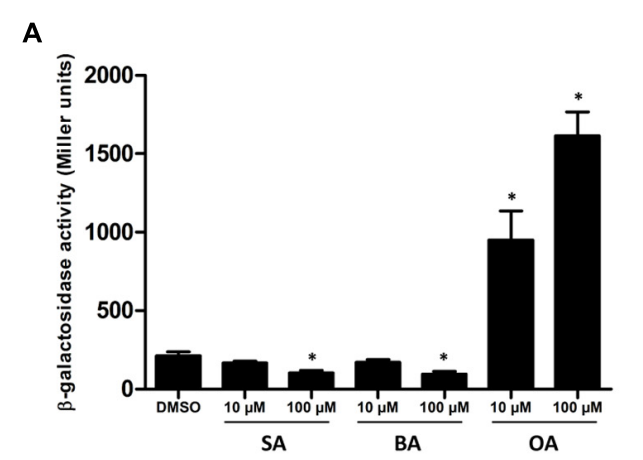

B

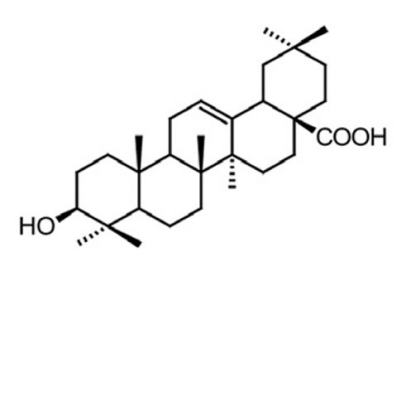

C

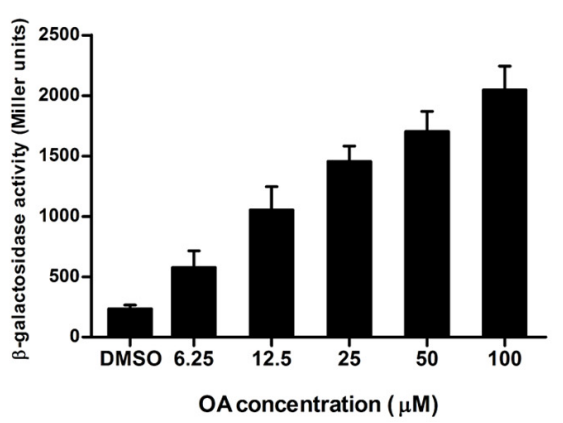

FIGURE 1 | Oleanolic acid (OA) induces T3SS expression of Ralstonia solanacearum in a concentration-dependent manner. (A) $\beta$-galactosidase activity of the popA-lacZYA reporter fusion gene in plant-sucrose (PS) medium supplemented with dimethyl sulfoxide (DMSO) or PS medium supplemented with low (10 $\mu$ M) or high $(100 \mu \mathrm{M})$ concentrations of stearic acid (SA), benzoic acid (BA) and oleanolic acid (OA). Values are representative of two independent experiments. Asterisks indicate that $\beta$-galactosidase activity after compound treatment is significantly lower or higher than that of the DMSO control $(P<0.05$, Student's $t$-test).

(B) Chemical structure of OA (Pollier and Goossens, 2012). (C) $\beta$-galactosidase activity of the popA-lacZYA reporter fusion gene in PS medium supplemented with DMSO or PS medium supplemented with different concentrations of OA. Columns represent the average $\beta$-galactosidase activity of three independent experiments; error bars indicate the standard deviation.

DMSO control (Figure 1C). The fold induction of $p o p A$ by $\mathrm{OA}$ at concentrations of 50,25 , and $12.5 \mu \mathrm{M}$ was $7.3,6.3$, and 4.5 , respectively. The expression of popA was induced by a 2.5 -fold of level when $6.25 \mu \mathrm{M}$ of $\mathrm{OA}$ was supplemented in the PS medium. The induction activity was decreased with the reduced $\mathrm{OA}$ concentration, suggesting that OA induced $p o p A$ expression in a concentration-dependent manner.

\section{OA Induces T3SS Expression Through the HrpG-HrpB Pathway}

In the previous experiments, we used a popA-lacZYA reporter strain to measure $p o p A$ expression and we found that $\mathrm{OA}$ induced the expression level of $p o p A$. In order to confirm this finding, we measured the mRNA level of popA after OA treatment by qRT-PCR. Compared to the DMSO control, a significantly higher level of popA mRNA was observed when the PS medium was supplemented with $100 \mu \mathrm{M}$ of OA (Figure 2). This result was consistent with the previous reporter strain based assay that showed that popA expression in $R$. solanacearum was induced by OA.

popA is a type III effector gene and its expression is directly controlled by $\mathrm{HrpB}$ whose expression is further regulated by HrpG, PrhG and other upstream regulators. Since the induction effect of $\mathrm{OA}$ on $p \circ p A$ expression has been validated, we want to know the effect of $\mathrm{OA}$ on the regulatory components of the T3SS which have been well described in $R$. solanacearum (Peeters et al., 2013b). The mRNA levels of genes in the T3SS signal pathway after DMSO or OA treatment were then measured. Our result showed that most of the T3SS upstream regulators were induced upon addition of $100 \mu \mathrm{M}$ of OA when compared to the DMSO control (Figure 3). The expression level of PrhG was not significantly affected after OA treatment. This suggests that the T3SS inducer OA induces the T3SS expression through the HrpG-HrpB pathway.

\section{OA Induces Only a Subset of Type III Effector Genes}

In $R$. solanacearum, $\mathrm{HrpB}$ is a downstream regulator in the T3SS signal cascade and it directly controls the transcription of type III effector genes. Based on the initial experiments, we found that OA altered the expression of both the T3SS downstream regulation gene $h r p B$ and the type III effector gene popA. To determine whether the induction of $h r p B$ by OA results in transcription activation of other effector genes, qRT-PCR was performed to examine the mRNA levels of other effector genes in the presence and absence of OA. Because $R$. solanacearum

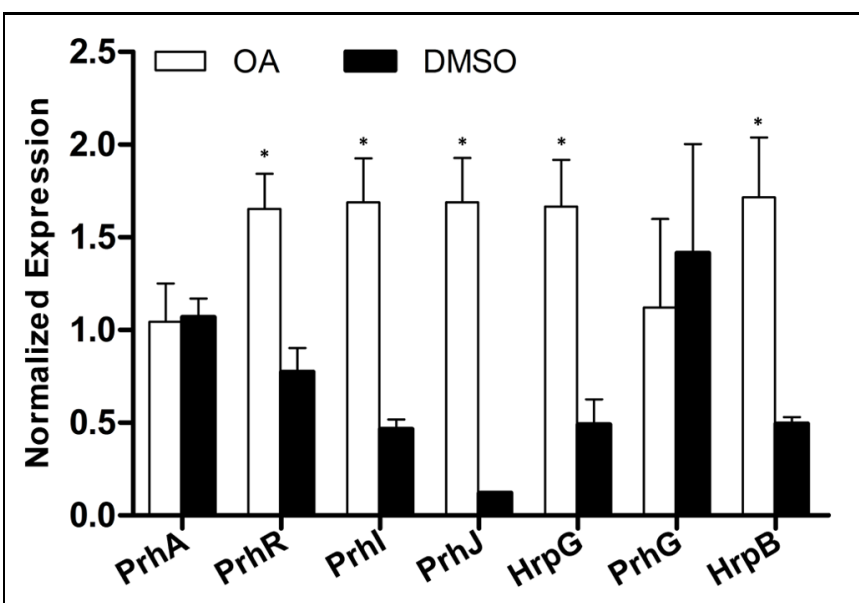

FIGURE 2 | Some but not all type III effector genes are induced by OA. The relative expression level of nine representative type III effector genes in PS medium supplemented with DMSO or $100 \mu \mathrm{M}$ of OA were determined by qRT-PCR. SerC was used as the reference gene to normalize the type III effector gene expression using the $\Delta \Delta \mathrm{Cq}$ method. Results are the average value of two biological replicates; error bars indicate the standard deviation. Asterisks indicate that these effector genes are significantly induced by $\mathrm{OA}$ compared to the DMSO control $(P<0.05$, Student's $t$-test). 


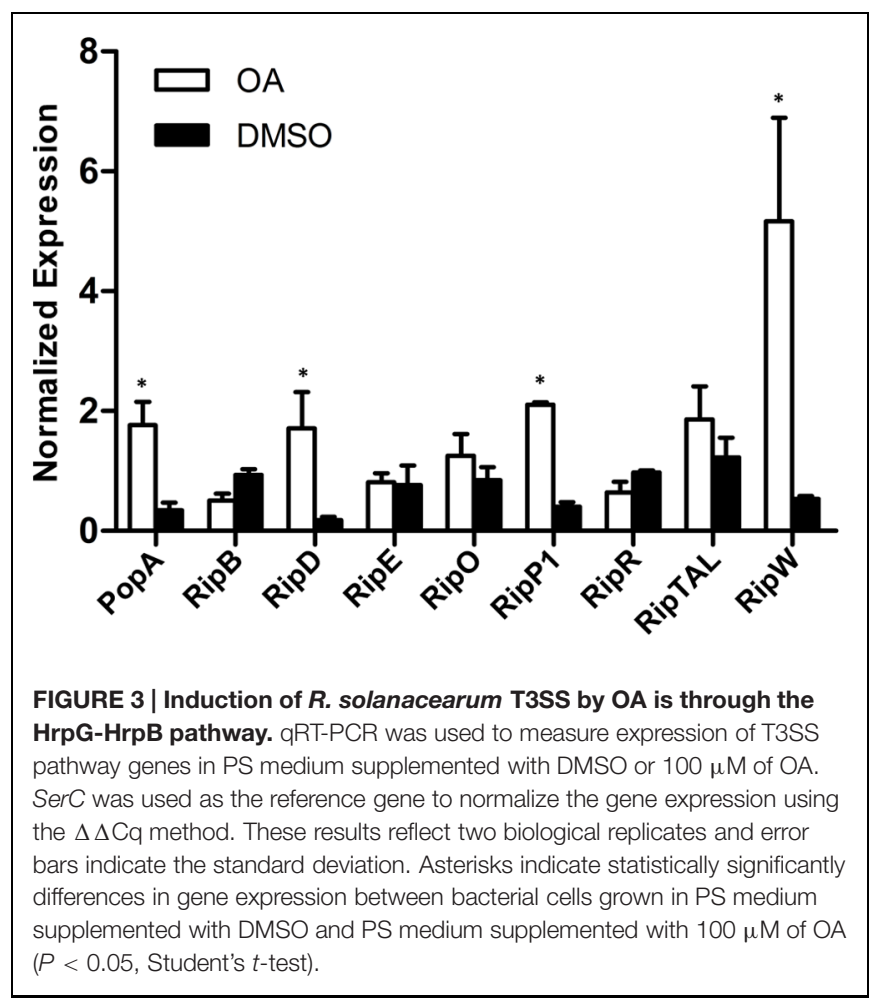

has a large repertoire of effectors and it is quite difficult to test the expression of all other effector genes, we chose eight representative effector genes for this experiment. The result

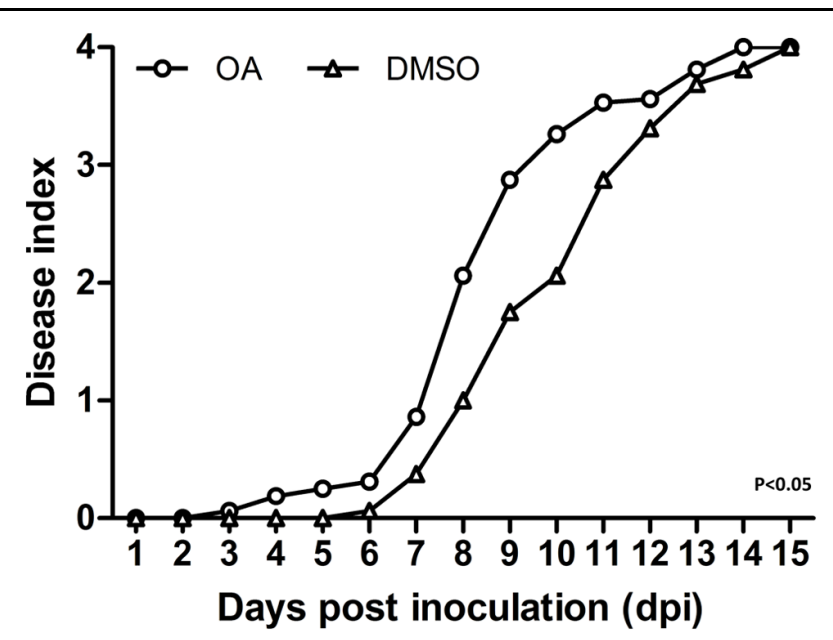

FIGURE 4 | Induction of T3SS by OA accelerates disease progress on tobacco. 6-week-old tobacco plants were inoculated with $R$. solanacearum treated with DMSO or $100 \mu \mathrm{M}$ of OA by pouring a bacterial suspension onto a pot containing an unwounded plant. Symptoms were rated daily using a disease index scale of 0-4 (0, no symptoms appeared; $1,1-25 \%$ of leaves wilted; 2, 26-50\% of leaves wilted; 3, 51-75\% of leaves wilted; 4, 76-100\% of leaves wilted). Each point represents the average disease index of 16 plants. The OA treatment was significantly different from the DMSO treatment $(P<0.05$; repeated-measures ANOVA). Similar results were observed in other two independent experiments. showed that five effector genes were similarly expressed in DMSO or OA treated cells (Figure 2). However, significantly higher levels of mRNA of another three effector genes, RipD, RipP1, and $R i p W$, were observed when the PS medium was supplemented with $100 \mu \mathrm{M}$ OA. This indicates that only a subset of type III effector genes were induced by the T3SS inducer OA.

\section{Other Virulence Factors in}

\section{R. solanacearum are not Affected by OA}

We hypothesize that T3SS inducers or inhibitors target only the bacterial T3SS, but not other virulence factors that play important roles in different infection stages. To test this, the growth rate of $R$. solanacearum after OA treatment was first determined. Since PS medium used for T3SS induction is a nutrition-poor medium and not able to support the growth of $R$. solanacearum over an $\mathrm{OD}_{600}$ of $0.2, \mathrm{~B}$ medium was selected to test the effect of $\mathrm{OA}$ on bacterial growth. In a $36 \mathrm{~h}$ growth curve, $100 \mu \mathrm{M}$ of OA did not cause a significant induction or inhibition on the growth of the bacterium (Supplementary Figure $\mathrm{S} 1$ ). Having shown that OA does not affect the R. solanacearum growth, we further tested whether this compound affects biofilm formation. A standard PVC microtiter plate assay was used to quantify $R$. solanacearum biofilm formation. The result showed that there was no significant difference in bacterial biofilm formation after treatment with DMSO or different concentrations of OA treatment (Supplementary Figure S2).

In $R$. solanacearum, the T3SS is linked with the quorum sensing system and the type II secretion system by the global virulence regulator PhcA (Mole et al., 2007). Given that OA significantly induced the expression of T3SS genes, we were interested in the effect of $\mathrm{OA}$ on the expression of other virulence regulation genes. To test this, we measured the mRNA level of the key regulator PhcA; quorum sensing regulators $\mathrm{PhcB}, \mathrm{PhcR}$, and $\mathrm{PhcS}$; and EPS secretion related genes $X p s R$ and EpsE. Although $\mathrm{OA}$ reduced the expression of $P h c A$ and several other genes, no significant inhibition was observed when evaluated with significance analysis at the level of $p=0.05$ (Supplementary Figure S3). It seems that OA does not affect the expression of other virulence regulation genes.

\section{OA Accelerates Disease Progress of Bacterial Wilt on Tobacco}

Based on the strong induction effect of OA on $R$. solanacearum T3SS, its ability to accelerate bacterial wilt disease progress was evaluated. In order to mimic the natural infection process, a soil soak virulence assay was used to measure the wilt disease progress of DMSO or OA treated bacteria on tobacco Yunyan87, the cultivar from which the original wild-type strain was isolated. Compared to the DMSO control, OA treated $R$. solanacearum accelerated the wilting of inoculated plants $[P<0.05$; repeatedmeasures analysis of variance (ANOVA)] (Figure 4). This experiment suggests that OA-mediated T3SS induction makes the bacteria more virulent on host plants. 


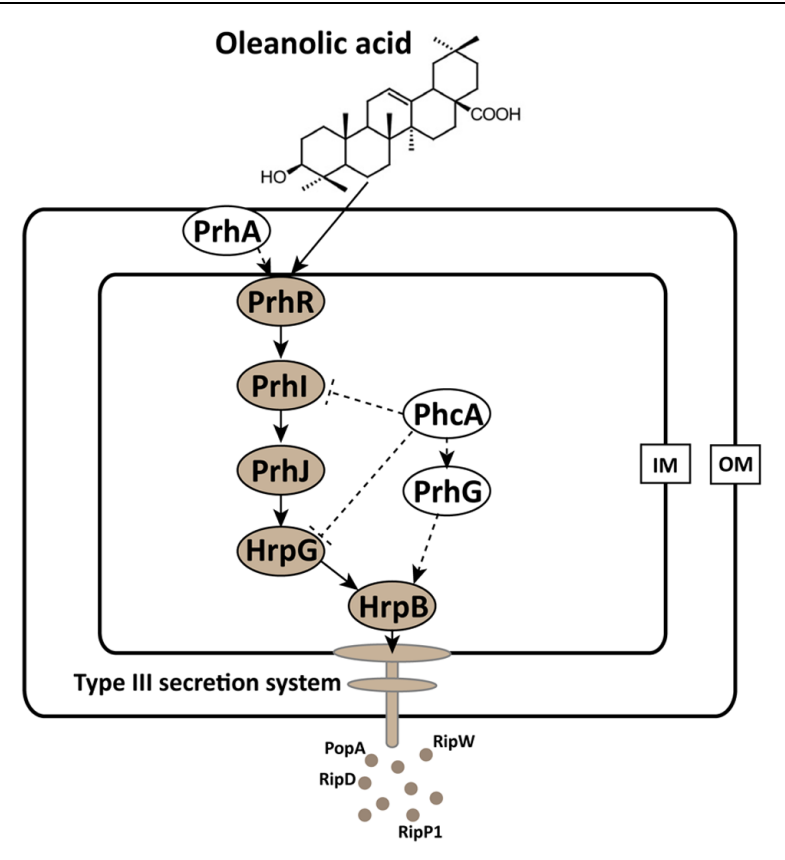

FIGURE 5 | Mode of OA on T3SS induction in R. solanacearum. The hrp gene expression in R. solanacearum is directly controlled by HrpB. HrpB is further controlled by two independent cascades PchA-PrhG and PrhA-PrhR/I-PrhJ-HrpG. The global regulator PhcA can also modulate T3SS expression through Prhl and HrpG (Peeters et al., 2013b). In this study, we observed that the T3SS inducer OA induced the type III effector gene expression (e.g., popA, RipD, RipW, and RipP1) through the HrpG-HrpB pathway. Ovals with gray background indicate regulators that induced by $O A$. Solid line arrows indicate the direct induction of these regulators by OA. IM, inner membrane; OM, outer membrane.

\section{DISCUSSION}

In this study, a total of 50 plant-derived compounds were tested for their activity on inhibiting or inducing the $R$. solanacearum T3SS expression. We found that 22 compounds significantly inhibited or induced the expression of the T3SS in this bacterium (Table 1), suggesting that plant-derived compounds are an abundant source of exogenous regulators of $R$. solanacearum T3SS. We were initially looking for T3SS inhibitors for this devastating plant pathogen and we did find some compounds (e.g., BA and SA) that are able to reduce the T3SS expression of this bacterium. However, the inhibitory effect of these compounds was not so pronounced. But interestingly, the compound OA strongly induced T3SS expression of R. solanacearum. Based on this observation, OA was chosen as an interesting candidate with which to investigate the mechanism of $R$. solanacearum T3SS regulation by exogenous compounds. Further experiments demonstrated that OA induced the T3SS through the HrpG-HrpB pathway and activated expression of some, but not all, type III effector genes. This T3SS inducer targeted only the T3SS without affecting other virulence determinants. In addition, induction of $R$. solanacearum T3SS by $\mathrm{OA}$ accelerated disease progress on tobacco.
Oleanolic acid is a widely distributed pentacyclic triterpenoid compound throughout the plant kingdom with several promising pharmacological activities, such as being an anti-inflammatory and antioxidant (Pollier and Goossens, 2012). It is interesting that OA was found to be able to induce T3SS expression of $R$. solanacearum in this study, suggesting that this special compound has a variety of biological functions in nature. Although T3SS induction by OA is beneficial for the pathogen, which does not comply with the promising pharmacological activity of OA, we here just regard it as a representative compound to investigate the mechanism of $R$. solanacearum T3SS regulation by exogenous compounds. It is in fact, not a surprise that the $R$. solanacearum T3SS was induced by OA. It has been shown that when pyruvate was supplemented as the carbon source in the medium, the level of $R$. solanacearum T3SS expression was induced (Arlat et al., 1992). More, previous studies have identified several plant phenolic compounds and derivatives as the T3SS inducers of $D$. dadantii, $P$. aeruginosa and E. amylovora (Yang et al., 2008; Yamazaki et al., 2012; Khokhani et al., 2013). These studies suggest that it is possible to induce the expression of the T3SS in diverse bacterial pathogens by plantderived compounds, which is consistent with our finding that the T3SS of $R$. solanacearum could be induced by OA. Some of the identified T3SS inducers are shown to have higher induction activity when used at high concentrations. In agreement with these observations, our data also showed that OA induced $R$. solanacearum T3SS expression in a concentration-dependent manner (Figure 1C). Since OA is a kind of plant-derived compound, we speculate that OA mimics the host environment and thus activates the T3SS expression. If this assumption is true, it may open new directions in chemical level for hostmicrobe interaction studies. That means, during the compatible interaction between susceptible hosts and pathogens, the host may secrete some compounds to induce the T3SS expression and favor the pathogen invasion. If it is true, identifying such compounds will deepen our current understanding of the hostmicrobe interaction.

The T3SS signal cascade has been well characterized in R. solanacearum. PrhA, an outer membrane receptor at the top of the $h r p$ regulatory pathway, is responsible for perceiving plant signals and activating the downstream regulators (Marenda et al., 1998; Aldon et al., 2000). The PrhA-dependent activation of T3SS is specific to bacteria-host contact but not required in medium (Marenda et al., 1998). In this study, we found that PS medium supplemented with OA induced downstream T3SS expression, but it did not induce the expression of the top regulator PrhA (Figure 3). There is a possibility that OA directly activates the PrhA protein at post-translational level but not transcriptional level. PrhI, PrhR, and PrhJ, the downstream regulators of PrhA, have also been shown to activate $h r p$ gene expression in response to contact with plant cells but not in the medium (Brito et al., 1999, 2002). It is worth noting that PrhI, PrhR, and PrhJ were induced by $\mathrm{OA}$ in our experiment. This suggests that although these regulators are not required for activation of hrp genes in medium, they can still be induced in medium. Another regulator in this cascade is HrpG, which is required for T3SS gene expression in minimal medium (Brito et al., 1999). Consistent 
with the previous study, our data demonstrated that hrpG expression was significantly induced by OA. In R. solanacearum, hrp gene expression in minimal medium is also controlled by PrhG which belongs to an independent pathway (Plener et al., 2010). In contrast with some other regulators, this regulator is required for T3SS gene activation in medium but not in the presence of plant cells. Interestingly, the medium-response regulator PrhG is not affected by OA. But PrhG showed a higher basal expression compared to other regulators. Together, our results suggest that the T3SS inducer OA affects the expression of T3SS through HrpG-HrpB pathway (Figure 5).

$R$. solanacearum has a large repertoire of type III effectors and homology of approximately one-third of effectors can be found in other bacterial plant pathogens (Peeters et al., 2013a; Deslandes and Genin, 2014). Although limited is known about the regulation of the effector genes expression in medium, an in planta transcriptome analysis has revealed that almost half of the effector genes are up-regulated in wilting tomato plants compared to expression in rich medium (Jacobs et al., 2012). This indicates firstly that type III effector production is still required at the later stages of bacterial wilt. Second, only a subset of effector genes from a given strain may contribute significantly to disease on a given host. Most importantly, the activation of $\mathrm{HrpB}$ does not mean that all downstream effector genes are also activated in a given environment, though $\mathrm{HrpB}$ is the master regulator of all the effector genes. In our study, we found that OA significantly induced $\operatorname{HrpB}$, but only three of eight tested effector genes were induced by OA (Figure 2). The $R$. solanacearum strain that we used in this study is originally isolated from tobacco. We do not know exactly how many effector genes our strain has, but based on the previous study (Jacobs et al., 2012), we guess only a subset of them are up-regulated in infected tobacco plants. The eight effectors we chose here are only representative conserved effector genes in $R$. solanacearum. We speculate that OA induced the expression of more effector genes if we test more and these induced effectors contribute to bacterial virulence. The later inoculation assay showed that $R$. solanacearum treated with $\mathrm{OA}$ is more virulent than $R$. solanacearum treated with DMSO (Figure 4), which may support the speculation that OA enhanced bacterial virulence by inducing the expression of some effector genes.

It is known that the T3SS is a vital pathogenicity factor in bacterial pathogens. Disruption of genes encoding structural components of the T3SS or upstream regulators always results in non-pathogenicity (Boucher et al., 1987). Based on this, researchers have tried to use $h r p$ - mutants that are defective in T3SS activation or translocation as potential bio-control agents to

\section{REFERENCES}

Aiello, D., Williams, J. D., Majgier-Baranowska, H., Patel, I., Peet, N. P., Huang, J., et al. (2010). Discovery and characterization of inhibitors of Pseudomonas aeruginosa type III secretion. Antimicrob. Agents Chemother. 54, 1988-1999. doi: 10.1128/AAC.015 98-09 control bacterial wilt (Frey et al., 1994), but this approach failed in field conditions. However, we can see from these studies that instead of producing hrp- mutants, inhibiting T3SS expression by using compounds would be an alternative option. Interestingly, our study identified a T3SS inducer. We also found that induction of the T3SS by this inducer accelerated the disease progress on tobacco, suggesting that if potential T3SS inhibitors exist, the T3SS inhibitor would be able to delay or even prevent disease occurrence.

In summary, this study screened a total of 50 plantderived compounds and identified that some of them could inhibit or induce the $R$. solanacearum T3SS expression. The mechanism of the representative T3SS inducer OA on regulation of $R$. solanacearum T3SS was further elucidated. These results suggest that plant-derived compounds are an abundant source of R. solanacearum T3SS regulators. Further screening may identify some strong T3SS inhibitors to disable the normal function of $R$. solanacearum T3SS providing an alternative strategy for the control of bacterial wilt.

\section{AUTHOR CONTRIBUTIONS}

Conceived and designed the experiments: WD performed the experiments: DW, YZ, XL, LY and WD. Analyzed the data: DW and WD wrote the paper: DW and WD.

\section{FUNDING}

This work was supported by a key project of the China National Tobacco Corporation (110201202002).

\section{ACKNOWLEDGMENT}

We are very grateful to Dr. Orlando de Lange for critical reading of the manuscript and helpful discussions. We also thank Elsevier for granting us permission and license to reuse the figure of the chemical structural of oleanolic acid (license number: 3766610157992).

\section{SUPPLEMENTARY MATERIAL}

The Supplementary Material for this article can be found online at: http://journal.frontiersin.org/article/10.3389/fmicb. 2015.01466

Aldon, D., Brito, B., Boucher, C., and Genin, S. (2000). A bacterial sensor of plant cell contact controls the transcriptional induction of Ralstonia solanacearum pathogenicity genes. EMBO J. 19, 2304-2314. doi: 10.1093/emboj/19.10.2304

Arlat, M., Gough, C. L., Zischek, C., Barberis, P. A., Trigalet, A., and Boucher, C. A. (1992). Transcriptional organization and expression of the large hrp gene cluster of Pseudomonas solanacearum. Mol. Plant. Microbe Interact. 5, 187-193. doi: 10.1094/MPMI-5-187 
Bailey, L., Gylfe, ^̊, Sundin, C., Muschiol, S., Elofsson, M., Nordström, P., et al. (2007). Small molecule inhibitors of type III secretion in Yersinia block the Chlamydia pneumoniae infection cycle. FEBS Lett. 581, 587-595. doi: 10.1016/j.febslet.2007.01.013

Boucher, C. A., Van Gijesgem, F., Barberis, P. A., Arlat, M., and Zischek, M. (1987). Pseudomonas solanacearum gene controling both pathogenicity and hypersensitivity on tobacco are clustered. J. Bacteriol. 169, 5626-5632.

Brito, B., Aldon, D., Barberis, P., Boucher, C., and Genin, S. (2002). A signal transfer system through three compartments transduces the plant cell contactdependent signal controlling Ralstonia solanacearum hrp genes. Mol. Plant. Microbe Interact. 15, 109-119. doi: 10.1094/MPMI.2002.15.2.109

Brito, B., Marenda, M., Barberis, P., Boucher, C., and Genin, S. (1999). PrhJ and hrpG, two new components of the plant signal-dependent regulatory cascade controlled by PrhA in Ralstonia solanacearum. Mol. Microbiol. 31, 237-251. doi: 10.1046/j.1365-2958.1999.01165.x

Cunnac, S., Occhialini, A., Barberis, P., Boucher, C., and Genin, S. (2004). Inventory and functional analysis of the large Hrp regulon in Ralstonia solanacearum: identification of novel effector proteins translocated to plant host cells through the type III secretion system. Mol. Microbiol. 53, 115-128. doi: 10.1111/j.13652958.2004.04118.x

Dahlgren, M. K., Kauppi, A. M., Olsson, M., Linusson, A., and Elofsson, M. (2007). Design, synthesis, and multivariate quantitative structure- activity relationship of salicylanilides potent inhibitors of type III secretion in yersinia. J. Med. Chem. 50, 6177-6188. doi: 10.1021/jm070741b

Deslandes, L., and Genin, S. (2014). Opening the Ralstonia solanacearum type III effector tool box: insights into host cell subversion mechanisms. Curr. Opin. Plant Biol. 20, 110-117. doi: 10.1016/.jpbi.2014.05.002

Duncan, M. C., Linington, R. G., and Auerbuch, V. (2012). Chemical inhibitors of the type three secretion system: disarming bacterial pathogens. Antimicrob. Agents Chemother. 56, 5433-5441. doi: 10.1128/AAC. 00975-12

Felise, H. B., Nguyen, H. V., Pfuetzner, R. A., Barry, K. C., Jackson, S. R., Blanc, M.-P., et al. (2008). An inhibitor of gram-negative bacterial virulence protein secretion. Cell Host Microbe 4, 325-336. doi: 10.1016/j.chom.2008.08.001

Frey, P., Prior, P., Marie, C., Kotoujansky, A., Trigalet-demery, D., Trigalet, A., et al. (1994). Hrp - Mutants of Pseudomonas solanacearum as potential biocontrol agents of tomato bacterial wilt Hrp- mutants of Pseudomonas solanacearum as potential biocontrol agents of tomato bacterial wilt. Appl. Environ. Microbiol. $60,3175-3181$.

Genin, S. (2010). Molecular traits controlling host range and adaptation to plants in Ralstonia solanacearum. New Phytol. 187, 920-928. doi: 10.1111/j.14698137.2010.03397.x

Genin, S., Brito, B., Denny, T. P., and Boucher, C. (2005). Control of the Ralstonia solanacearum Type III secretion system (Hrp) genes by the global virulence regulator PhcA. FEBS Lett. 579, 2077-2081. doi: 10.1016/j.febslet.2005.0 2.058

Jacobs, J. M., Babujee, L., Meng, F., Milling, A., and Allen, C. (2012). The in planta transcriptome of Ralstonia solanacearum: conserved physiological and virulence strategies during bacterial wilt of tomato. MBio 3:e114-112. doi: 10.1128/mBio.00114-12.Editor

Jacobs, J. M., Milling, A., Mitra, R. M., Hogan, C. S., Ailloud, F., Prior, P., et al. (2013). Ralstonia solanacearum requires PopS, an ancient AvrE-family effector, for virulence and to overcome salicylic acid-mediated defenses during tomato pathogenesis. MBio 4:e875-13. doi: 10.1128/mBio.00875-13

Jones, J. D. G., and Dangl, J. L. (2006). The plant immune system. Nature 444, 323-329. doi: 10.1038/nature05286

Khokhani, D., Zhang, C., Li, Y., Wang, Q., Zeng, Q., Yamazaki, A., et al. (2013). Discovery of plant phenolic compounds that act as type III secretion system inhibitors or inducers of the fire blight pathogen, Erwinia amylovora. Appl. Environ. Microbiol. 79, 5424-5436. doi: 10.1128/AEM.00845-13

Li, Y., Hutchins, W., Wu, X., Liang, C., Zhang, C., Yuan, X., et al. (2015). Derivative of plant phenolic compound inhibits the type III secretion system of Dickeya dadantii via HrpX/HrpY two-component signal transduction and Rsm systems. Mol. Plant Pathol. 16, 150-163. doi: 10.1111/mpp.12168

Li, Y., Peng, Q., Selimi, D., Wang, Q., Charkowski, A. O., Chen, X., et al. (2009). The plant phenolic compound p-coumaric acid represses gene expression in the Dickeya dadantii type III secretion system. Appl. Environ. Microbiol. 75, 1223-1228. doi: 10.1128/AEM.02015-08
Marenda, M., Brito, B., Callard, D., Genin, S., Barberis, P., Boucher, C., et al. (1998). PrhA controls a novel regulatory pathway required for the specific induction of Ralstonia solanacearum hrp genes in the presence of plant cells. Mol. Microbiol. 27, 437-453. doi: 10.1046/j.1365-2958.1998. 00692.x

Mole, B. M., Baltrus, D. A., Dangl, J. L., and Grant, S. R. (2007). Global virulence regulation networks in phytopathogenic bacteria. Trends Microbiol. 15, 363371. doi: 10.1016/j.tim.2007.06.005

Monteiro, F., Genin, S., van Dijk, I., and Valls, M. (2012). A luminescent reporter evidences active expression of Ralstonia solanacearum type III secretion system genes throughout plant infection. Microbiology 158, 2107-2116. doi: 10.1099/mic.0.058610-0

Negrea, A., Bjur, E., Ygberg, S. E., Elofsson, M., Wolf-Watz, H., and Rhen, M. (2007). Salicylidene acylhydrazides that affect type III protein secretion in Salmonella enterica serovar typhimurium. Antimicrob. Agents Chemother. 51, 2867-2876. doi: 10.1128/AAC.00223-07

Occhialini, A., Cunnac, S., Reymond, N., Genin, S., and Boucher, C. (2005). Genome-wide analysis of gene expression in Ralstonia solanacearum reveals that the hrpB gene acts as a regulatory switch controlling multiple virulence pathways. Mol. Plant. Microbe Interact. 18, 938-949. doi: 10.1094/MPMI-180938

Peeters, N., Carrère, S., Anisimova, M., Plener, L., Cazalé, A.-C., and Genin, S. (2013a). Repertoire, unified nomenclature and evolution of the Type III effector gene set in the Ralstonia solanacearum species complex. BMC Genomics 14:859. doi: 10.1186/1471-2164-14-859

Peeters, N., Guidot, A., Vailleau, F., and Valls, M. (2013b). Ralstonia solanacearum, a widespread bacterial plant pathogen in the post-genomic era. Mol. Plant Pathol. 14, 651-662. doi: 10.1111/mpp.12038

Plener, L., Manfredi, P., Valls, M., and Genin, S. (2010). PrhG, a transcriptional regulator responding to growth conditions, is involved in the control of the type III secretion system regulon in Ralstonia solanacearum. J. Bacteriol. 192, 1011-1019. doi: 10.1128/JB.01189-09

Pollier, J., and Goossens, A. (2012). Oleanolic acid. Phytochemistry 77, 10-15. doi: 10.1016/j.phytochem.2011.12.022

Schell, M. A. (2000). Control of virulence and pathogenicity genes of Ralstonia solanacearum by an elaborate sensory network. Annu. Rev. Phytopathol. 38, 263-292. doi: 10.1146/annurev.phyto.38.1.263

Stauber, J. L., Loginicheva, E., and Schechter, L. M. (2012). Carbon source and cell density-dependent regulation of type III secretion system gene expression in Pseudomonas syringae pathovar tomato DC3000. Res. Microbiol. 163, 531-539. doi: 10.1016/j.resmic.2012.08.005

Tang, X., Xiao, Y., and Zhou, J.-M. (2006). Regulation of the type III secretion system in phytopathogenic bacteria. Mol. Plant-Microbe Interact. 19, 11591166. doi: 10.1094/MPMI-19-1159

Tree, J. J., Wang, D., McInally, C., Mahajan, A., Layton, A., Houghton, I., et al. (2009). Characterization of the effects of salicylidene acylhydrazide compounds on type III secretion in Escherichia coli O157:H7. Infect. Immun. 77, 4209-4220. doi: 10.1128/IAI.00562-09

Van Dijk, K., Fouts, D. E., Rehm, A. H., Hill, A. R., Collmer, A., and Alfano, J. R. (1999). The Avr (effector) proteins HrmA (HopPsyA) and AvrPto are secreted in culture from Pseudomonas syringae pathovars via the Hrp (type III) protein secretion system in a temperature- and $\mathrm{pH}$-sensitive manner. J. Bacteriol. 181, 4790-4797.

Veenendaal, A. K. J., Sundin, C., and Blocker, A. J. (2009). Small-molecule type III secretion system inhibitors block assembly of the shigella type III secreton. J. Bacteriol. 191, 563-570. doi: 10.1128/JB.01004-08

Wei, Z., Sneath, B. J., and Beer, S. V. (1992). Expression of Erwinia amylovora hrp genes in response to environmental stimuli. J. Bacteriol. 174, 1875-1882.

Wengelnik, K., and Bonas, U. (1996). HrpXv, an AraC-type regulator, activates expression of five of the six loci in the hrp cluster of Xanthomonas campestris pv. vesicatoria. J. Bacteriol. 178, 3462-3469.

Yamazaki, A., Li, J., Zeng, Q., Khokhani, D., Hutchins, W. C., Yost, A. C., et al. (2012). Derivatives of plant phenolic compound affect the type III secretion system of Pseudomonas aeruginosa via a GacS-GacA two-component signal transduction system. Antimicrob. Agents Chemother. 56, 36-43. doi: 10.1128/AAC.00732-11

Yang, F., Korban, S. S., Pusey, P. L., Elofsson, M., Sundin, G. W., and Zhao, Y. (2014). Small-molecule inhibitors suppress the expression of both type III 
secretion and amylovoran biosynthesis genes in Erwinia amylovora. Mol. Plant Pathol. 15, 44-57. doi: 10.1111/mpp.12064

Yang, S., Peng, Q., San Francisco, M., Wang, Y., Zeng, Q., and Yang, C. H. (2008). Type III secretion system genes of Dickeya dadantii 3937 are induced by plant phenolic acids. PLoS ONE 3:e2973. doi: 10.1371/journal.pone.0002973

Yao, J., and Allen, C. (2007). The plant pathogen Ralstonia solanacearum needs aerotaxis for normal biofilm formation and interactions with its tomato host. J. Bacteriol. 189, 6415-6424. doi: 10.1128/JB.00398-07

Yoshimochi, T., Zhang, Y., Kiba, A., Hikichi, Y., and Ohnishi, K. (2009). Expression of hrpG and activation of response regulator $\mathrm{HrpG}$ are controlled by distinct signal cascades in Ralstonia solanacearum. J. Gen. Plant Pathol. 75, 196-204. doi: 10.1007/s10327-009-0157-1

Zhang, Y., Chen, L., Yoshimochi, T., Kiba, A., Hikichi, Y., and Ohnishi, K. (2013). Functional analysis of Ralstonia solanacearum PrhG regulating the hrp regulon in host plants. Microbiol. (United Kingdom) 159, 1695-1704. doi: 10.1099/mic.0.067819-0

Zhang, Y., Kiba, A., Hikichi, Y., and Ohnishi, K. (2011). prhKLM genes of Ralstonia solanacearum encode novel activators of hrp regulon and are required for pathogenesis in tomato. FEMS Microbiol. Lett. 317, 75-82. doi: 10.1111/j.15746968.2011.02213.x

Zhang, Y., Luo, F., Wu, D., Hikichi, Y., Kiba, A., Igarashi, Y., et al. (2015). PrhN, a putative marR family transcriptional regulator, is involved in positive regulation of type III secretion system and full virulence of Ralstonia solanacearum. Front. Microbiol. 6:357. doi: 10.3389/fmicb.2015.0 0357

Conflict of Interest Statement: The authors declare that the research was conducted in the absence of any commercial or financial relationships that could be construed as a potential conflict of interest.

Copyright ( $\odot 2015 \mathrm{Wu}$, Ding, Zhang, Liu and Yang. This is an open-access article distributed under the terms of the Creative Commons Attribution License (CC BY). The use, distribution or reproduction in other forums is permitted, provided the original author(s) or licensor are credited and that the original publication in this journal is cited, in accordance with accepted academic practice. No use, distribution or reproduction is permitted which does not comply with these terms. 